\section{Des mots}

et des maux

Que peut-on espérer

des récits de vie

dans la relation de soin

à travers les âges?

Marie Gaille

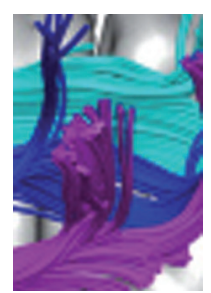

CERSES, centre de recherche sens, éthique, société ; UMR 8137 CNRS - université Paris Descartes, 45 , rue des Saints-Pères, 75006 Paris, France. mariegaille@yahoo.fr
Jacques Quintin (bureau de développement de l'éthique, université de Sherbrooke, Québec, Canada), philosophe et psychiatre de formation, a organisé et animé en mai 2011 , dans le cadre du $79^{e}$ congrès de I'ACFAS (Association canadienne-française pour l'avancement des sciences), un colloque intitulé «Des mots et des maux : que peut-on espérer des récits de vie dans la relation de soin à travers les âges ? » [1]. L'intérêt d'une telle manifestation est qu'elle se place délibérément au carrefour de plusieurs approches du soin dans la pensée bioéthique et entend les confronter les unes aux autres. Au centre de son questionnement se situe ce que l'on nomme l'éthique narrative (narrative ethics). Ce courant de pensée, apparu outre-Atlantique dans les années 1980, participe de ce qu'on appelle communément un «tournant narratif » manifeste dans les sciences humaines et sociales, mais aussi, dans une moindre mesure, dans la conception des cursus de formation en faculté de médecine.

Encore peu connue en France, l'éthique narrative pourrait cependant trouver sa place dans la formation des médecins, dans le cadre d'une réforme qui cherche, depuis quelques années, à donner plus de place aux sciences humaines et sociales et à la réflexion éthique. Elle consiste notamment dans l'étude d'œuvres littéraires qui offrent des représentations élaborées de l'expérience de la maladie et de l'approche de la mort. Elle se déploie aussi dans le recueil des témoignages de patients pour déboucher sur celui de l'expérience des équipes médicales. Elle se nourrit enfin de récits de situations thérapeutiques élaborés par des acteurs extérieurs au soin, mais mobilisés en contexte hospitalier, précisément pour tenir compte du vécu du patient et de la dimension éthique de nombre de décisions médicales (par exemple, voir [2]).

Au sujet de la relation entre le patient et le médecin, le développement de l'éthique narrative relève initialement d'une ambition précise: il s'agit à travers elle de restituer à la pratique médicale une dimension humaniste qu'on estime souvent dévoyée, voire perdue, dans une pratique où prime le recours aux biotechnologies et aux données probantes. Par la suite, l'éthique narrative a nourri plusieurs finalités. Il s'agit de donner aux médecins les outils pour une meilleure écoute du patient, et de les orienter vers une véritable prise en compte du point de vue de ce dernier et de son vécu de la maladie. II convient également de faire du consentement du patient autre chose qu'un accord formel à telle ou telle proposition thérapeutique : une décision ancrée dans les convictions propres du patient et réellement partagée avec son médecin. Enfin, le recours à l'éthique narrative est conçu comme un outil du diagnostic lui-même, le récit du patient pouvant contribuer, à travers les éléments qu'il livre au médecin, à comprendre la pathologie dont il est atteint. La dimension subjective de la santé est mise en valeur par ce courant éthique, mais pas de n'importe quelle manière puisque cette dimension se manifeste avant tout par le récit de soi : les patients, lorsqu'ils racontent leur vécu et leur parcours dans la maladie, comprennent et montrent aux équipes médicales que le rétablissement passe certes par l'usage des ressources de la médecine moderne, mais aussi par l'invention d'une autre façon de voir les choses et de les nommer. Selon l'expression de l'un des intervenants, Patrick Vinay (université de Montréal, hôpital Notre-Dame, Montréal), néphrologue, il existe une « narrativité guérissante ». Se raconter peut susciter de la souffrance, mais permet aussi de faire face à la fragilisation identitaire suscitée par la maladie (on ne peut plus faire ce que I'on effectuait autrefois sans peine, on devient dépendant des autres pour les gestes plus quotidiens) et ainsi sauver le désir de vivre. 
Selon J. Quintin, qui s'inscrit dans le sillage des œuvres de P. Ricoeur et de J.-F. Malherbe, le médecin peut contribuer à l'émergence de cette narrativité, en encourageant le patient à lui livrer son expérience de vie, en l'aidant à atténuer l'incertitude et l'insuffisance des mots, en instaurant un dialogue qui éclaire le patient sur ce qui l'anime et le maintient vivant, malgré la maladie. Pour ce faire, le médecin se place dans la meilleure position possible vis-à-vis du patient s'il accepte de reconnaître qu'il a lui aussi sa propre histoire. Le récit de vie du patient qui s'élabore dans leur dialogue résulte de la rencontre de deux biographies. Comme l'indique H. Doucet (université de Montréal), qui a prononcé la conférence d'ouverture de ce colloque, l'utilité clinique de l'éthique narrative ne se borne pas au processus de rétablissement du patient. Lorsque des prises en charge médicales n'ont pas abouti ou se sont «mal passées », aux dires mêmes des équipes, elle peut être mobilisée de façon fructueuse dans le cadre de consultations a posteriori : les médecins y sont invités à expliciter les raisons pour lesquelles, selon eux, les choses ne se sont pas déroulées de façon souhaitable. En ce sens, l'éthique narrative contribue à une compréhension des choses qui rend possible une reprise de l'action médicale harmonieuse, apaisée et éclairée par son expérience passée.

Sans conférer à l'éthique narrative plus de puissance qu'elle n'en a pour améliorer le soin médical, sans nier les chausse-trappes et les ambivalences du récit de soi, ce colloque entend promouvoir une réflexion utile de part et d'autre de l'océan atlantique sur la place à donner à la narration dans l'enseignement médical, les pratiques de soin et la réflexion éthique sur celles-ci. Ce congrès a témoigné qu'il restait encore beaucoup de travail à accomplir en ce sens, dans un contexte tant francophone qu'anglophone. Quels genres de récit sont produits en contexte thérapeutique, dans le cadre d'une décision médicale ? Quelle part de «vérité » leur accorder? Et jusqu'à quel point la « vérité » est-elle l'enjeu de l'éthique narrative? Quel poids accorder au récit dans la réflexion éthique? Dans quelle limite, surtout pour ne pas créer une situation dissymétrique entre des situations où le patient peut s'exprimer et se trouve écouté, et d'autres où le patient n'a pas de voix (comme le nouveau-né), une voix discordante (comme un patient dément) ou une voix qui a perdu sa légitimité (comme un patient qu'on ne juge plus compétent) ?

The contribution of narrative ethics to healthcare

\section{CONFLIT D'INTÉRÊTS}

L'auteur déclare n'avoir aucun conflit d'intérêts concernant les données publiées dans cet article.

\section{RÉFÉRENCES}

1. ACFAS. $79^{e}$ congrès de l'Association francophone pour le savoir. Université de Sherbrooke, Québec, Canada, 7-12 mai 2011.

2. Zaner R. Conversations on the edge. Narratives of ethics and illness. Washington DC : Georgetown University Press, 2004.

\section{Collection L'Actualité Chimique-Livres}

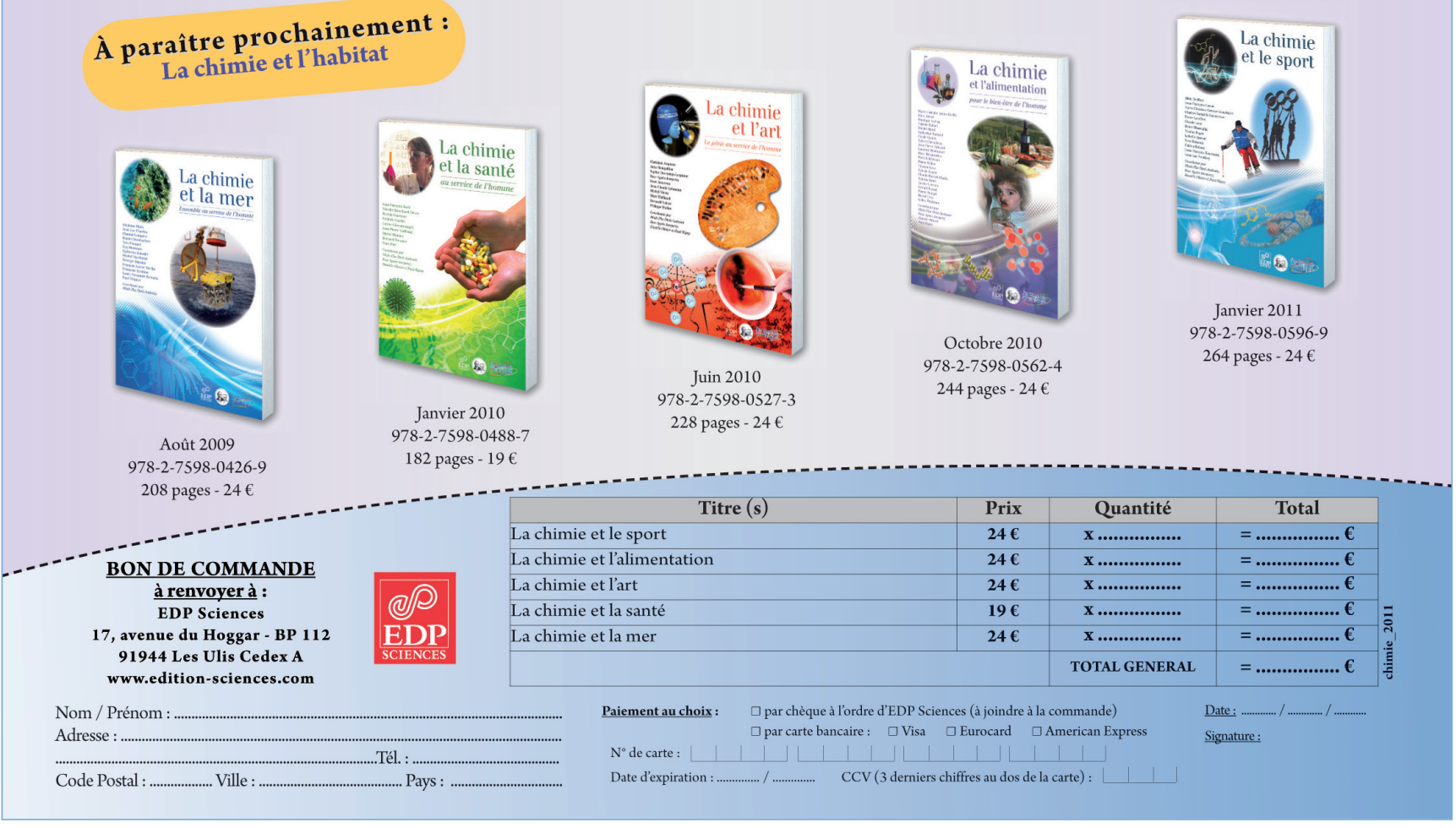

